\title{
Particle Filter Target Tracking Algorithm Based on MCMC Iteration Cubature
}

\author{
Song Gao, Yenan Liu and Chaobo Chen \\ School of Electronic Information Engineering \\ Xi'an Technological University, Xi'an 710021, China \\ gaos@xatu.edu.cn
}

\begin{abstract}
In view of the problem of particle degradation and tracking accuracy in the standard particle filter tracking target algorithm, a new improved particle filter algorithm called Iterated Cubature Kalman Particle Filter (ICKPF) is proposed in this paper. The new ICKPF algorithm is based on the Markov Chain Monte Carlo (MCMC), and the cubature rule based on numerical integration method is used to calculate the mean and covariance, which generates the proposal distribution for the particle filter. The current measurements are integrated into the proposal distribution. Therefore, degree of approximation to the system posterior density is improved. Simulation results show that the estimation error of the ICKPF-MCMC algorithm is much better than other algorithms.
\end{abstract}

Keywords: target tracking; particle filter; iterated Cubature Kalman filter; Markov Chain Monte Carlo

\section{Introduction}

There is a large number of non-linear and non- Gaussian systems that have state estimation problems in the field of target tracking, failure detection and navigation guidance. Most conventional filtering algorithms are based on the Kalman theory framework which is useless to non-linear systems. Gordon ${ }^{[1]}$ is the one who first apply the particle filter (Particle Filter, PF) algorithm to non-linear and non-Gaussian state estimation. PF algorithm is one of the non-linear filtering algorithms which is based on Bayesian theory and Monte Carlo method. It is widely used in the areas of target tracking application for it is not affected by non-linear and non-Gaussian problems. Standard particle filter regards state transition function as the prior distribution of particles, and the influence of current measurement to the state estimates will not be considered. There is a great deviation between the samples from the importance of sampling density and the true posterior probability density, which can cause degradation phenomena and filter divergence [2]. Therefore, the key issue to improve the standard particle filter literature is to select the importance density function. The extended Kalman filter (EKF) is used to generate the importance density function in reference [3], the filtering precision is improved, but the EKF in the process of the model linearization caused too much error. Reference [4] proposed unscented Kalman particle (Unscented Kalman Particle Filter, UPF) algorithm to generate the distribution function. The accuracy of UPF algorithm state estimation is higher than EPF algorithm, so that we can get a better filtering effect only if the UPF sigma point is set reasonably. Arasaratnam and other people [8-9] proposed the Cubature Kalman Particle Filter algorithm (Cubature Kalman Particle Filter CKPF) based on Cubature Kalman (Cubature Kalman Filter, CKF). CKPF algorithm computes the non-linear random variable of the average of the values and covariance with cubature value principle points. It integrated into the current measurement information and made the proposed distribution of particle filter closer to the posterior probability distribution. 
The filter performance is greatly improved, and it has a strong real-time performance [10-11].

For the target tracking with a strong non-linear state estimation problems, this paper uses the proposal distribution of particle filter algorithm generated by iterative cubature Kalman particle filter algorithm (ICKPF) in literature[12] and integrates statistical theory of Markov Chain Monte Carlo (Markov Chain Monte Carlo, MCMC) method. It combines ICKF algorithms with typical MCMC algorithms-MH (Metropolis Hastings) sampling algorithm [13-14] to solve the problem of particle degeneration and make the sampling particle more diversified, and then improving the tracking accuracy.

\section{Standard Particle Filter Algorithm (PF)}

$\mathrm{PF}$ is an approximate number solution algorithm based on Monte Carlo methods and Bayesian estimation, which provides an approximate Bayesian solution to the recursive filtering problem in discrete time, the basic idea is to construct a sample based posterior probability density function, the sample mean is adopted to take place of the integral calculation based on posterior probability density function, then the minimum variance estimation is obtained.

Suppose, the nonlinear discrete-time dynamic system is expressed by formula (1):

$$
\left\{\begin{array}{c}
x_{k+1}=f\left(x_{k}\right)+w_{k} \\
z_{k}=h\left(x_{k}\right)+v_{k}
\end{array}\right.
$$

Where, the $x_{k}$ and $z_{k}$ are system state vector and measured value at time $k$, $p\left(x_{0}\right)$ is a known initial state probability distribution density, and $f(\cdot)$ and $h(\cdot)$ represent the system state transition model and measurement model function. $w_{k}$ and $v_{k}$ are uncorrelated zero-mean process noise and measurement noise, which covariance is $Q_{k}$ and $R_{k}$ respectively.

The idea of Particle Filter is to use a particle collection $\left\{x_{0: k}^{i}, w_{k}^{i}\right\}_{i=1}^{N}$ to indicate a posteriori probability density function $p\left(x_{0: k} \mid z_{0: k}\right)$ of system target status $x_{k}$ at $k$ time, in which $w_{k}^{i}$ represents the corresponding weight of particle $x_{0: k}^{i}$, and meet $\sum_{i=1}^{N} w_{k}^{i}=1 . \quad x_{0: k}=\left\{x_{i}, i=0, \ldots, k\right\}$ is the system status collection at moment $k$, and the posterior probability density function of target status at moment $\mathrm{k}$ can be approximately expressed as follows:

$$
p\left(x_{0: k} \mid z_{1: k}\right) \approx \sum_{i=1}^{N} w_{k}^{i} \delta\left(x_{0: k}-x_{0: k}^{i}\right)
$$

Where, $\delta(\cdot)$ is the Delta function? Seed collection $\left\{x_{0: k}^{i}, w_{k}^{i}\right\}_{i=1}^{N}$ can be sampled from the importance distribution function $q\left(x_{0: k} \mid z_{1: k}\right)$ based on importance sampling theory. Weight update equation can be written as follows:

$$
w_{k}^{i} \propto w_{k-1}^{i} \frac{p\left(z_{k} \mid x_{k}^{i}\right) p\left(x_{k}^{i} \mid x_{k-1}^{i}\right)}{q\left(x_{k}^{i} \mid x_{0: k-1}^{i}, z_{1: k}\right)}
$$

The main steps [15] of the standard particle filter is described:

(1). Initialization

Generate particles from the prior $p\left(x_{0}\right)$ to obtain particle collection 


$$
\left\{x_{0: k}^{i}, w_{k}^{i}\right\}_{i=1}^{N} \text {. }
$$

(2). Sample

For $\mathrm{k}=1, \ldots, \mathrm{T}$

For $\mathrm{i}=1, \ldots, \mathrm{N}$

Sample $x_{k}^{i}$ from the proposal distribution $q\left(x_{0: k} \mid z_{1: k}\right)$.

(3). Weighting

Evaluate particle weights up to a normalizing constant:

$$
w_{k}^{i} \propto w_{k-1}^{i} \frac{p\left(z_{k} \mid x_{k}^{i}\right) p\left(x_{k}^{i} \mid x_{k-1}^{i}\right)}{q\left(x_{k}^{i} \mid x_{0: k-1}^{i}, z_{1: k}\right)}
$$

Normalize the particle weights:

$$
w_{k}^{i}=\frac{w_{k}^{j}}{\sum_{j=1}^{N} w_{k}^{j}}, i=1, \ldots, N
$$

(4).Output

Output a set of particle $\left\{x_{0: k}^{i}, w_{k}^{i}\right\}_{i=1}^{N}$ that can be used to approximate the posterior distribution $p\left(x_{k} \mid z_{1: k}\right) \approx \sum_{i=1}^{N} w_{k}^{i} \delta\left(x_{0: k}-x_{0: k}^{i}\right)$.

(5).Resample

Resample the particles $\left\{x_{k}^{i}, w_{k}^{i}\right\}$ with probabilities $w_{k}^{i}$ to obtain $\mathrm{N}$ i.i.d random particles $\left\{\tilde{x}_{k}^{i}, 1 / N_{N}\right\}$, approximately distributed according $p\left(x_{k} \mid z_{1: k}\right)$. End

End

One of the main problems of standard PF algorithm is the particle degradation, after a few iterations, there are only very few particles left behind whose weight is big. Resampling is an effective way to solve the problem of degeneration. When degradation is obvious, resampling is added on the basis of importance sampling to eliminate the particle with low weight and remain the particle with high weight to inhibit degradation. Relying on resample with a request that the design of distribution should approach to the probability density function as far as possible, a good proposal distribution will improve the particle degradation and depleted problem.

\section{Iterative Cubature Kalman Particle Filter (ICKPF)}

Iterative cubature Kalman particle filter [12] is the algorithm which introduces the Gauss-Newton iteration algorithm to the Cubature Kalman particle filter algorithm. In order to construct the proposed distribution function of particle filter, the algorithm using the current measurement information and cubature value points to calculate the average and the variance of the non-linear random function directly by setting a defined group of points and corresponding weights .So that the proposal particles become closer to real samples, the steps are as follows: 
1. Initialization: when $k=0$, the corresponding variance $P_{0}^{i}$ and the sampling particles $\left\{x_{0}^{i}, i=1,2, \ldots, N\right\}$ which come from the priori probability $p\left(x_{0}\right)$ should be initialized.

2. Loop iterations for $\mathrm{k}=1: \mathrm{T}$

Updating sampling particles, $\mathrm{i}=1,2, \ldots \mathrm{N}, \mathrm{n}=1,2, \ldots$, Niter according to the iterative cubature Kalman algorithm every moment.

(1) Time updating

Calculating the cubature points:

$$
x_{j, k-1}^{i}=s_{k-1}^{i} \varepsilon_{j}+\hat{x}_{k-1}^{i}
$$

The $S_{k-l}^{i}$ is the square root of the probability distribution variance $P_{k-1}^{i}$ at the $k-1$ moment. $\varepsilon_{j}$ is the cubature point, $j=1,2, \ldots, M, M$ represents the total cubature points which is twice the dimension of the status, and $M=2^{n_{x}[10]}$. The cubature is transferred through the non-linear state equation.

$$
x_{j, k}^{* i}=f\left(x_{j, k-1}^{i}\right)
$$

Cubature points spread by nonlinear equation of state:

$$
\begin{gathered}
\hat{x}_{k}^{i}=\frac{1}{M} \sum_{j=1}^{M} x_{j, k}^{* i} \\
P_{k}^{i}=\frac{1}{M} \sum_{j=1}^{M} x_{j, k}^{* i} x_{j, k}^{* i}-\hat{x}_{k}^{i} \hat{x}_{k}^{i^{T}}+Q_{k-1}
\end{gathered}
$$

(2) Measurement updating

For $\mathrm{n}=1$ : Niter

A new factorization and cubature points are generated which can be expressed by formula (8) and (9).

$$
\begin{gathered}
s_{k}^{i,(n)}=\operatorname{chol}\left(P_{k}^{i(n)}\right) \\
x_{j, k}^{i,(n)}=s_{k}^{i,(n)} \varepsilon_{j}+\hat{x}_{k}^{i,(n)}
\end{gathered}
$$

The $\mathrm{n}$-th iteration of the states and the variance estimation can be calculated by formula (10).

$$
z_{j, k}^{i,(n)}=h\left(x_{j, k}^{i,(n)}\right)
$$

Then, the measurement forecast, the new interest rate variance and covariance estimation are given by formula (11), (12) and (13).

$$
\begin{gathered}
\hat{z}_{k}^{i,(n)}=\frac{1}{M} \sum_{j=1}^{M} z_{j, k}^{i,(n)} \\
P_{z, k}^{i,(n)}=\frac{1}{M} \sum_{j=1}^{M} z_{j, k}^{i,(n)} z_{j, k}^{i,(n)^{T}}-\hat{z}_{k}^{i,(n)} \hat{z}_{k}^{i,(n)^{T}}+R_{k} \\
P_{x z, k}^{i,(n)}=\frac{1}{M} \sum_{j=1}^{M} x_{j, k}^{i,(n)} z_{j, k}^{i,(n)^{T}}-\hat{x}_{k}^{i,(n)} \hat{z}_{k}^{i,(n)^{T}}
\end{gathered}
$$

In addition, the gain, states and covariance estimation are calculated by formula (14), (15) and (16).

$$
K_{k}^{i,(n)}=P_{x z, k}^{i,(n)} P_{z z, k}^{i,(n)^{-1}}
$$




$$
\begin{gathered}
\hat{x}_{k}^{i,(n+1)}=\hat{x}_{k}^{i,(n)}+K_{k}^{i,(n)}\left[z_{k}-h\left(\hat{x}_{k}^{i,(n)}\right)-P_{x z, k}^{i,(n)^{T}} P_{k}^{i^{-1}}\left(\hat{x}_{k}^{i}-\hat{x}_{k}^{i,(n)}\right)\right] \\
P_{k}^{i,(n+1)}=P_{k}^{i}-K_{k}^{i,(n)} P_{z z, k}^{i,(n)} K_{k}^{i,(n)^{T}}
\end{gathered}
$$

End.

3. Generating the importance density function.

$$
q\left(x_{k}^{i} \mid x_{k-1}^{i}, z_{1: k}\right)=N\left(\hat{x}_{k}^{i,(n)}, P_{k}^{i,(n)}\right)
$$

4. Calculating a normalized weight.

$$
\omega_{k}^{i}=\widetilde{\omega}_{k}^{i} / \sum_{i=1}^{N} \widetilde{\omega}_{k}^{i}
$$

5. Resampling, obtaining the same weight of $1 / N$ of the particles set.

$\left\{x_{k}^{i}, p_{k}^{i}, i=1,2, \cdots, N\right\}$

6. Outputing status and variance estimation at $\mathrm{k}$ time.

$$
\begin{gathered}
x_{k}=\frac{1}{N} \sum_{i=1}^{N} \hat{x}_{k}^{i} \\
P_{k}=\frac{1}{N} \sum_{i=1}^{N} \omega_{k}^{i}\left(x_{k}-\hat{x}_{k}^{i}\right)\left(x_{k}-\hat{x}_{k}^{i}\right)^{T}
\end{gathered}
$$

The ICKPF algorithm, which generates the proposed distribution of particle filter, is obtained by combining ICKF algorithm with PF algorithm in this section. And the current measurement information is able to integrate into the algorithm to improve filtering effect.

\section{Iteration Cubature Kalman Particle Filter based on MCMC}

\subsection{MCMC Moving Steps}

The basic idea of resampling is to copy particles with big weights and eliminate particles with small weights. While, particles with big weights are repeatedly copied after several times of sampling, it will lead to particle-depleted problems and reduce the diversity of the particles. Then, the posterior probability distribution of the system can't be represented adequately.

Markov Chain Monte Carlo (MCMC) [13] algorithm can solve particles depleted problem effectively. MCMC algorithm uses memory less property of random simulation methods of the Markov Chain. Its basic idea is: constructing a Markov Chain and it has the same distribution $\pi$. When the Markov Chain number of iterations $n \rightarrow \infty$, the $X_{n}$ distribution can get infinitely close to $\pi$. Then, we can get a random sample of approximately obey P's based on this method.

This paper used the Metropolis-Hasting $(\mathrm{MH})$ algorithm which is a stochastic simulation method based on the theory of Markov Chains .The specific steps are as follows:

Step 1: Choosing initial state of Markov Chain $x_{0}=x$ arbitrarily;

Step 2: Generating a movement of $x^{\prime}$ by the transfer of kernel $q(\cdot \mid x)$;

Step 3: Generating $u(0,1)$ random number $u$, if $u \leq \alpha\left(x, x^{\prime}\right)$, then $x_{1}=x^{\prime}$, otherwise keep the current status unchanged, i.e. $x_{1}=x_{0}=x^{\prime}$;

Step 4: Repeating the above steps, generate $x_{2}, x_{3}, \cdots, x_{n}$ in turn. 


\subsection{The Iterative Cubature Particle Filter Combined MCMC Algorithm}

The idea based on MCMC iterative Cubature Kalman Particle Filter algorithm uses ICKF to generate proposed distribution function, the $N\left(\hat{x}_{k}^{i}, P_{k}^{i}\right)$ is adopted to update the mean and variance of the particles. Then the values will integrate into the Metropolis-Hasting sampling algorithm after the resampling of ICKPF algorithm to increase the diversity of the particles and improve filtering accuracy.

MCMC algorithm generates the sample to obey target distribution by constructing Markov Chain. Assuming the particles $\left\{\hat{x}_{0: k}^{i}\right\}$ obey to a posterior probability density distribution function $p\left(\hat{x}_{0: k}^{i} \mid z_{1: k}\right)$, we can use a stationary distribution of $P\left(x_{0: k} \mid z_{1: k}\right)$ Markov Chain transfer Kernel for each particle $\left\{\hat{x}_{0: k}^{i}\right\}$. We can get the following function:

$$
\int \alpha\left(x_{0: k} \mid \hat{x}_{0: k}^{i}\right) p\left(\hat{x}_{0: k}^{i} \mid z_{1: k}\right) d \hat{x}_{0: k}=P\left(x_{0: k} \mid z_{1: k}\right)
$$

Then the new particles $\left\{\hat{x}_{0: k}^{i}\right\}$ still obey to the same posterior probability density distribution. In the process of iteration, when importance sampling combined with MCMC, the particle can move to a different place so as to avoid depleted phenomenon of the particle.

The $\mathrm{MH}$ algorithm which commonly used the MCMC is integrated into the resampling of the ICKF algorithm; we can get the new algorithm ICKPF-MCMC.

Specific implementation steps are as follows:

1 . We can get threshold $u$ by sampling from the interval $[0,1]$ in accordance with uniform distribution, i.e., $u \sim U[0,1]$, updating particle state and variance through the ICKF algorithm. Sample particles are updated through the iterative Cubature Kalman algorithm each time, $\mathrm{i}=1,2 \ldots \mathrm{N}, \mathrm{n}=1,2 \ldots$ Niter

(1) Update Time

The forecast estimated $x_{k}^{i^{*}}$ and forecast variances $p_{k}^{i^{*}}$ are calculated through formula (4) to (7).

(2) Measurements updating

Measurement updating is an iterative process with $x_{k}^{i^{*}}$ and $p_{k}^{i^{*}}$ as the initial value. Suppose, the n-th iteration and the variance estimate are $\hat{x}_{k}^{i,(n)^{*}}$ and $P_{k}^{i,(n)^{*}}$, $\mathrm{n}=0,1,2 \ldots$ Niter. Then, new factorization and cubature points are generated, which expressed by the following formula.

$$
\begin{gathered}
s_{k}^{i,(n)^{*}}=\operatorname{chol}\left(P_{k}^{i,(n)^{*}}\right) \\
x_{j, k}^{i,(n)^{*}}=s_{k}^{i,(n)^{*}} \varepsilon_{j}+\hat{x}_{k}^{i,(n)^{*}}
\end{gathered}
$$

And the n-th iteration of the status and the variance estimation can be calculated by formula (22), (23) and (24).

$$
\begin{aligned}
& K_{k}^{i,(n)^{*}}=P_{x z, k}^{i,(n)^{*}}\left(P_{z z, k}^{i,(n)^{*}}\right)^{-1} \\
& \hat{x}_{k}^{i,(n+1)^{*}}=\hat{x}_{k}^{i,(n)^{*}}+K_{k}^{i,(n)^{*}}\left[z_{k}-h\left(\hat{x}_{k}^{i,(n)^{*}}\right)-P_{x z}^{\left.i,(n)^{*}\right)^{T}} P_{k}^{i^{*-1}}\left(\hat{x}_{k}^{i^{*}}-\hat{x}_{k}^{i,(n)^{*}}\right)\right] \\
& P_{k}^{i,(n+1)^{*}}=P_{k}^{i^{*}}-K_{k}^{i,(n)^{*}} P_{z z, k}^{i,(n)^{*}} K_{k}^{i,(n)^{* T}}
\end{aligned}
$$

Where, $P_{x z, k}^{i,(n)^{*}}$ and $P_{z z, k}^{i,(n)^{*}}$ are generated by formula (12) and (13). 
2. Sampling

$$
x_{k}^{i^{*}} \sim q\left(x_{k}^{i^{*}} \mid x_{0: k}^{i^{*}}, z_{1: k}\right)=N\left(x_{k}^{i,(n)^{*}}, P_{k}^{i,(n)^{*}}\right)
$$

3. When the following condition comes into existence.

Then, the move is accepted:

$$
u \leq\left\{1, \frac{p\left(z_{k} \mid x_{k}^{i^{*}}\right) p\left(x_{k}^{i^{*}} \mid \hat{x}_{k-1}^{i}\right) q\left(\hat{x}_{k}^{i} \mid \hat{x}_{0: k-1}^{i}, z_{1: k}\right)}{p\left(z_{k} \mid \hat{x}_{k}^{i}\right) p\left(\hat{x}_{k}^{i} \mid \hat{x}_{k-1}^{i}\right) q\left(x_{k}^{i^{*}} \mid \hat{x}_{0: k-1}^{i}, z_{1: k}\right)}\right\}
$$

Otherwise, the move will refused:

$$
x_{0: k}^{i}=\left(\hat{x}_{0: k-1}^{i}, x_{k}^{i^{*}}\right), p_{0: k}^{i}=\left(p_{0: k-1}^{i}, p_{k}^{i^{*}}\right)
$$

4. Repeat steps 1,2 , and 3.

$$
x_{0: k}^{i}=\hat{x}_{0: k}^{i}, p_{0: k}^{i}=p_{0: k}^{i}
$$

\section{Simulation Results and Analysis}

In order to verify the efficiency of target tracking by using the improved algorithm, the system state space model of the literature [10] is adopted to conduct simulation. Meanwhile, the simulation of the extended Kalman particle filter(EPF) algorithm, the extended Kalman particle filter(EPF-MCMC) algorithm based on MCMC, the unscented Kalman particle filter(UPF) algorithm, the unscented Kalman particle filter(UPF-MCMC) algorithm based on MCMC, iterative cubature Kalman particle filter (ICKPF) algorithm and the standard particle filter(PF) algorithm has been done. The simulation results are analyzed and compared.

The state space model of non-linear system is established:

$$
\begin{gathered}
x_{k}=1+\sin [\omega \pi(k-1)]+0.5 x_{k-1}+w_{k-1} \\
z_{k}=\left\{\begin{array}{l}
0.2 x_{k}^{2}+v_{k}, k \leq 30 \\
0.5 x_{k}-2+v_{k}, k>30
\end{array}\right.
\end{gathered}
$$

Where, $\omega=0.04$, the process noise obey to Gamma distribution, and $w_{k} \sim \gamma(3,2)$. Observing noise is obey to Gaussian distribution, and $v_{k} \sim N(0,0.0001)$. The initial state $x_{0}=1$, the initial variance $P_{0}=0.75$, the number of particles $N=200$, the times of observations $T=60$. After 50 times Monte Carlo simulations, the simulation results are as follows.

Figure 1 and figure 2 show the state estimation curve respectively generated by the PF, EPF, EPF-MCMC, UPF, UPF-MCMC, ICKPF and ICKPF-MCMC algorithms when the number of particles is 200. In Table 1, it shows these five algorithms' RMSE mean, variance and average running time comparison when the number of the particle is 200 . 


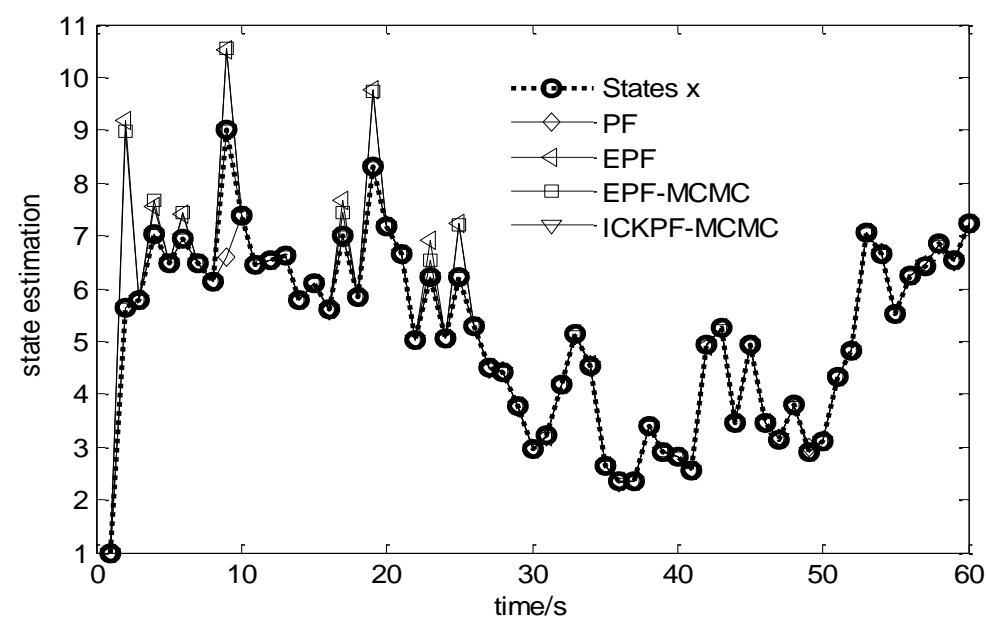

Figure 1. The State Estimation Curve of PF, EPF, EPF-MCMC and ICKPF-MCMC

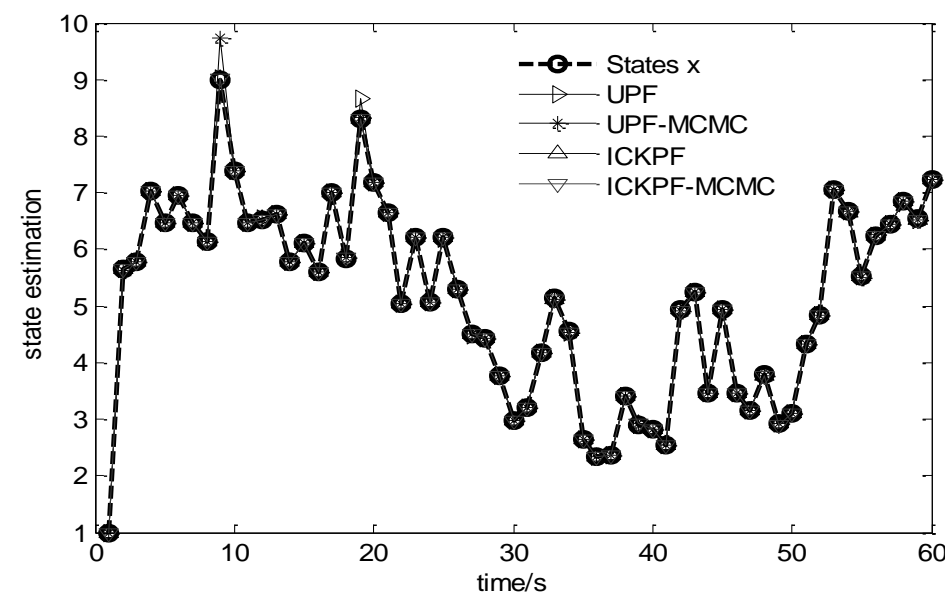

Figure 2. The State Estimation Surve of UPF, UPF-MCMC, ICKPF and ICKPF-MCMC Algorithms

From figure 1 and figure 2, it can be seen that the non-linear of observation equation are weak after 30 steps; as a result, each filtering algorithm has better tracking performance. The ICKPF and ICKPF-MCMC algorithms obtain the best performance in the first 30 steps of the strong nonlinear filtering tracking. But in table 1, it can be seen that the estimation accuracy and tracking performance of ICKPF-MCMC algorithm will be better.

Table 1. The Filtering Algorithms are of Value to the Variance $(N=200)$

\begin{tabular}{|c|c|c|c|}
\hline Algorithm & All RMSE value & RMSE variance & Run Time/s \\
\hline PF & 0.4795 & 0.056538 & 0.50518 \\
\hline EPF & 0.33595 & 0.017554 & 0.91014 \\
\hline EPF-MCMC & 0.32151 & 0.015284 & 1.9808 \\
\hline UPF & 0.084596 & 0.0082117 & 3.2318 \\
\hline UPF-MCMC & 0.070297 & 0.0077119 & 6.7436 \\
\hline ICKPF & 0.0054594 & $6.0173 E-7$ & 3.4341 \\
\hline ICKPF-MCMC & 0.0052301 & $3.2993 \mathrm{E}-7$ & 7.5459 \\
\hline
\end{tabular}


Table 1 shows that the estimation error of the ICKPF-MCMC algorithm accounts for only about $1.09 \%, 1.62 \%, 1.63 \%, 6.19 \%, 7.45 \%$ and $95.8 \%$ of the standard PF algorithm, the EPF algorithm, the EPF-MCMC algorithm, the UPF algorithm, the UPF-MCMC algorithm and the algorithm ICKPF respectively.

From table 1 and figure 3 and figure 4, it can be seen that under the same conditions, the ICKPF-MCMC algorithm takes the state estimates of updated measurement as a reference when constructing importance density function, and optimizes the proposed distribution to increase the diversity of particles through MCMC algorithm. It makes the state estimation more accurate and effective .The more effective samples, the higher estimation accuracy. In contrast EPF-MCMC and UPF-MCMC algorithm, computing time is somewhat long, but the tracking accuracy is improved.

In the process of state estimation, the current measured value is used to adjust the tracing error in the ICKPF-MCMC algorithm, then, bigger error can be avoided appearing in the process of estimation.

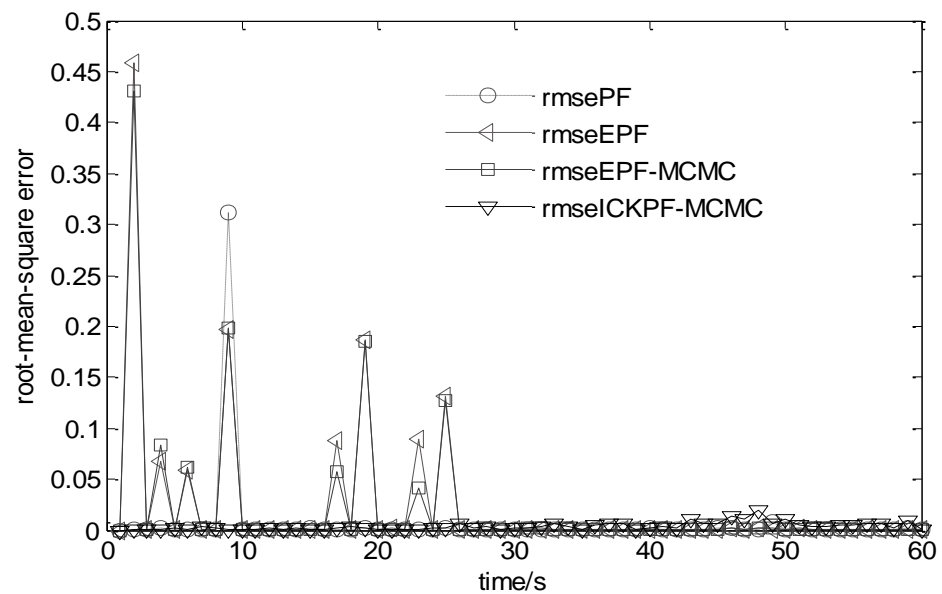

Figure 3. RMS Error Curve of PF, EPF, EPF-MCMC and ICKPF-MCMC Algorithms

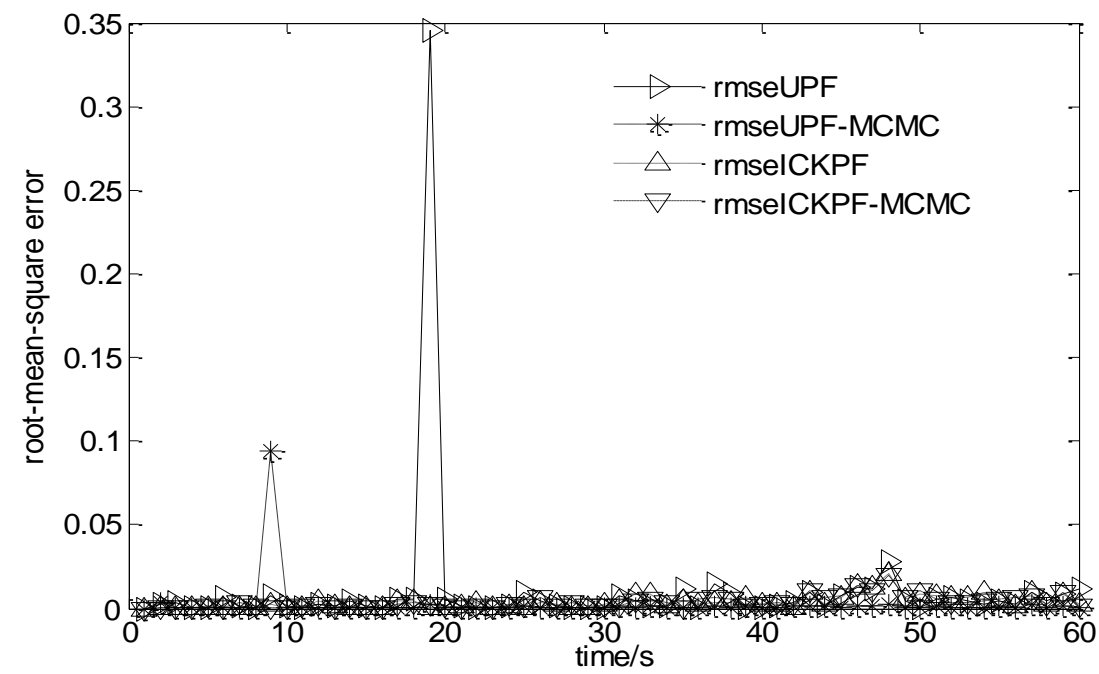

Figure 4. RMS Error Curve of UPF, UPF-MCMC, ICKPF and ICKPF-MCMC Algorithms 


\section{Conclusion}

The iterative cubature Kalman particle filter based on MCMC is put forward in this paper, and a recommended distribution function of particle filter is produced by using the ICKF, then the current measurement information can be integrated into the distribution function and the result is more consistent with real status of the importance density function. Meanwhile, MCMC algorithm is introduced to overcome the degradation problem of particles; it increases the diversity of effective particles. Simulation experiments and the results showed that the algorithm is significantly better than the standard particle filter algorithms, extended Kalman particle filter algorithm and MCMC extended Kalman algorithm. And compared with Unscented Kalman particle filter and the MCMC unscented Kalman particle filter, its running time increased slightly, but the accuracy is improved, and the filter effect is also improved compared with iterative Cubature Kalman particle filter.

\section{Acknowledgements}

This work is supported by the National Natural Science Foundation of China (61271362), the Shaanxi International Science and Technology Cooperation Project (2015KW-024) and the Weapon Pre-research Fund (62201070317).

\section{References}

[1] N J. Gordon, D J. Salmond and A F M. Smith, "Novel approach to nonlinear/non-Gaussian Bayesian state estimation [C]", IEE Proceedings F (Radar and Signal Processing). IET Digital Library, vol. 140, no. 2, (1993), pp. 107-113.

[2] S Y. Cheng and J Y. Zhang, "Review on particle filters [J]", Journal Of Astronautics, vol. 29, no. 4, (2008), pp. 1099-1111.

[3] N. De Freitas, M. Niranjan and A H. Gee, "Sequential Monte Carlo methods to train neural network models [J]", Neural Computation [J], vol. 12, no. 4, (2000), pp. 955-993.

[4] R. Van der Merwe, A. Douce and N. de Freitas, "The unscented particle filter [R]", Cambridge, UK: Engineering Departme, Cambridge University, (2000), pp. 1-45.

[5] W. Guo, C. Han and M. Lei, "Particle distribution control for an iterated unscented Kalman particle filter [J]”, Journal of Tsinghua University (Science and Technology), vol. 47, (2007), pp. 1866-1869.

[6] X X. Wang, Q. Pan and H. Huang, "Overview of deterministic sampling filtering algorithms for nonlinear system [J]", Control and Decision, vol. 27, no. 6, (2012), pp. 801-812.

[7] Y. Wu, D. Hu and M. Wu, "A numerical integration perspective on Gaussian filters [J]", IEEE Transactions on Signal Processing, vol. 54, no. 8, (2006), pp. 2910-2921.

[8] I. Arasaratnam and S. Haykin, "Cubature Kalman filters [J]", IEEE Transactions on Automatic Control, vol. 54, no. 6, (2009), pp. 1254-1269.

[9] I. Arasaratnam, S. Haykin and T R. Hurd, "Cubature Kalman filtering for continuous-discrete systems: Theory and simulations [J]”, IEEE Transactions on Signal Processing, vol. 58, no. 10, (2010), pp. 4977-4993.

[10] J. Mu, Y. Cai and J. Zhang, "Cubature particle filter and its application [J]", Journal of Xi'an Jiaotong University, vol. 45, no. 8, (2011).

[11] F. Sun and L J. Tang, "Cubature particle filter [J]", Systems Engineering and Electronics, vol. 33, no. 11, (2011), pp. 2554-2557.

[12] WHJZZ. Wenquan and Q. Baoping, "Iterated Cubature Kalman particle filter algorithm [J]", Journal of Southeast Univer $\neg$ sity (Natural Science Edition), (2013), S1.

[13] C. Andrieu, P M. Djurić and A. Doucet, "Model selection by MCMC computation [J]", Signal Processing, vol. 81, no. 1, (2001), pp. 19-37.

[14] S. Godsill and T. Clapp, "Improvement strategies for Monte Carlo particle filters [M]", Sequential Monte Carlo Meth $\neg$ ods in Practice. Springer New York, (2001), pp. 139-158.

[15] P. Li, T. Zhang and A E C. Pece, "Visual contour tracking based on particle filters [J]", Image and Vision Computing, vol. 21, no. 1, (2003), pp. 111-123. 


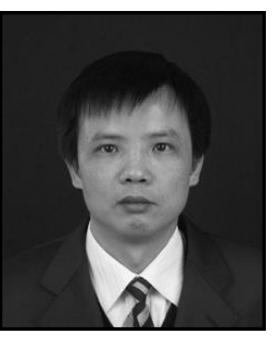

\section{Authors}

Song Gao, he received the bachelor of engineering at the of automatic control from Huazhong University of Science and Technology, Wuhan, China, in 1988, the master degree of engineering in automation from department of automation, Northwestern Polytechnical University, Xi'an, China, in 1995 and the Ph.D degree in automation from college of automation, Northwestern Polytechnical University, Xi'an, China, in 2011. He is mainly engaged in the research of intelligent control, multi-source information fusion and complex network control. His research interests include Bayesian estimation and sequential Monte Carlo methods.

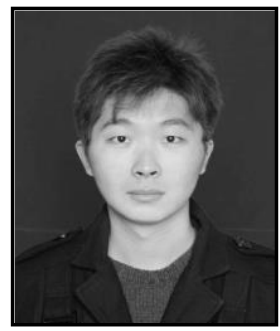

Yenan Liu, he received the bachelor of engineering at School of Electronic Information Engineering Xi'an Technological University, Xi'an, China, in 2011. Now he is a postgraduate student. He is mainly engaged in the research of intelligent control and multi-source information fusion.

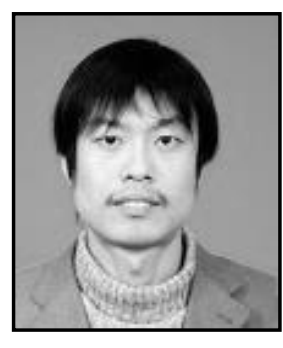

Chaobo Chen, he received the bachelor of engineering at School of Electronic Information Engineering Xi'an Technological University, Xi'an, China, in 2001 and the master degree of engineering in control theory and control engineering from Xi'an Technological University, Xi'an, China, in 2009.

His research interests include intelligent control, multi-source information fusion, independent decision-making and complex network control direction. 
International Journal of Signal Processing, Image Processing and Pattern Recognition Vol.8, No.9 (2015) 